\title{
Takayasu arteritis in a patient presenting with stroke and improvement of hemiparesis following vascular bypass surgery: A case report
}

\author{
ZOLTAN GALAJDA ${ }^{1}$, FELICIAN STANCIOIU ${ }^{1}$, DEMETRIOS A. SPANDIDOS ${ }^{2}$, GEORGE LAZOPOULOS ${ }^{3}$, \\ MIHAI CUCU $^{1}$, EUGEN QENDRO ${ }^{1}$, MARIUS FLOROIU ${ }^{1}$ and STELIOS KTENIADAKIS ${ }^{3}$ \\ ${ }^{1}$ Angiomedica Hospital, 020657 Bucharest, Romania; ${ }^{2}$ Laboratory of Clinical Virology, \\ University of Crete Medical School; ${ }^{3}$ Department of Cardio-Thoracic Surgery, \\ University Hospital of Heraklion, University of Crete Medical School, 71409 Heraklion, Greece
}

Received February 12, 2020; Accepted April 7, 2020

DOI:10.3892/wasj.2020.44

\begin{abstract}
Takayasu arteritis (TA) is a rare disease, with an estimated incidence in general population of $<1 /$ year $/$ million, and its presentation as a stroke is an even less common occurrence. The case presented herein is unusual for the fact that the surgical procedure was performed on an urgent basis, irrespective of the active or inactive status of the disease; the bypass technique involved multiple sites; the patient was administered an original combination of medications before and after the procedure, and the patient exhibited significant neurological improvement after the subacute period of the stroke (6 weeks after stroke, 1 week after the procedure), which was sustained at the 18-month follow-up. Published literature on bypass surgery for TA indicate that the majority of complications (aneurysms, stenosis, intracerebral hemorrhage, etc.) occur during the early stages and affect approximately one-third of patients; this procedure was also successful as the patient subsequently began recovering motor function, indicating the successful, sustained re-vascularization of the affected motor areas. Being able to restore the patient's independent activities of daily living is an important goal, which was achieved in this patient and may serve as a reference point for what is possible to achieve in these cases.
\end{abstract}

\section{Introduction}

Takayasu arteritis (TA, also known as 'pulseless disease') was first described in 1908 by the Japanese ophthalmologist, Mikito Takayasu, in the retinal vessels of a patient. Currently, it is known that this rare type of vasculitis (incidence, $0.3-4 /$ million/year)

Correspondence to: Dr Felician Stancioiu, Angiomedica Hospital, Strada Cosminului 13, 020657 Bucharest, Romania

E-mail: felicians@bio-forum.net

Key words: Takayasu arteritis, vascular bypass in vasculitis, recovery after stroke predominantly affects young females and is commonly associated with tenderness of the carotid artery, arm claudication, ocular disturbances, weakening of pulses, differences in blood pressure between arms, syncope and other central nervous system symptoms (1). The diagnostic criteria for TA, published in 1990 by the American College of Rheumatology (2), have a sensitivity of $91 \%$ and specificity of $95 \%$ and suggest the presence of at least 3 of the following: i) An age of $<40$ years; ii) claudication of the extremities; iii) decreased brachial artery pulse; iv) differences of $>10 \mathrm{mmHg}$ in systolic blood pressure between arms; v) bruit over subclavian/aorta; and vi) arteriographic abnormalities (3).

The underlying pathological processes in TA consist of vascular wall inflammation, immune dysfunction via the expression of human leukocyte antigen (HLA) antigens on vascular endothelial cells (4), particularly in patients with HLA-B*52, and the reduced number of regulatory $\mathrm{T}$ (Treg) cells (5), conjunctive tissue deterioration [activation of matrix metalloproteinases (MMP)2, 3 and 9 (6), the increased expression of vascular cell adhesion molecule-1 (VCAM-1), e-Selectin and intercellular cell adhesion molecule-1 (ICAM-1)] (7), and the stimulation of platelet aggregation. The activation of leukocyte nuclear factor (NF)- $\kappa \mathrm{B}$ is followed by the secretion of pro-inflammatory cytokines, such as interferon- $\alpha$ (IFN- $\alpha$ ), tumor necrosis factor- $\alpha$ (TNF- $\alpha$ ), and interleukin (IL)-6, IL-8, IL-17A and IL-18; the serum levels of these cytokines (IL-6, IL-12 and IL-18) are associated with disease activity (1). The histopathology of TA-affected blood vessels, more often observed in specimens obtained by post-surgical excision and autopsies than biopsy, is characterized by granulomatous inflammation, particularly in the vasa vasorum and the medio-adventitial junction, edema, the infiltration of mononuclear cells, laminar necrosis, and the deterioration of elastic fibers and smooth muscle cells (3).

The trigger for vascular inflammation seems to be the presence of circulating bacterial antigens and the subsequent immune cross-reaction involving vascular endothelial cells $(4,8)$. There is a known molecular mimicry between the human and mycobacterial $65-\mathrm{kDa}$ heat shock protein (HSP) and this molecule has been found in aortic biopsies (vasa 
vasorum and media) from patients with TA. Moreover, patients with TA have serum IgG antibodies against aortic endothelial cells directed against mycobacterial and human 60-65 kDa HSP (9), and T cells are also active against mycobacterial and human 65-kDa HSP (1). Apart from mycobacterium tuberculosis, other pathogens may be involved in TA; elevated plasma levels of pentraxin-related protein (PTX-3) are observed both in TA and in infections with Aspergillus fumigatus, Neisseria meningitidis and Pseudomonas aeruginosa (10).

The treatment of patients with TA aims to suppress both systemic and vascular inflammation with either corticosteroids, methotrexate, azathioprine, mycophenolate mofetil, cyclophosphamide or leflunomide $(11,12)$. In refractory cases there are good results with biological agents, antagonists of TNF- $\alpha$ and the Il-6 receptor (IL-6R), such as ustekinumab, infliximab, etanercept, adalimumab, rituximab, tocilizumab, and in pregnant patients treated with certolizumab pegol (13). However, TA has also been observed after the initiation of treatment with anti-TNF- $\alpha$ agents, and thus far, two patients with spondyloarthritis, one patient with rheumatoid arthritis, and one patient with Crohn's disease who developed TA following treatment with anti-TNF- $\alpha$ agents have been reported (13). Notably, the natural stilbenoid, curcumin (14), and resveratrol (15) have been shown to exert favorable effects on TA, probably due to their anti-TNF- $\alpha$ actions and their promoting effects on circulatory Treg functions. Finally, minocycline, a MMP inhibitor, in combination with steroids, has also been shown to be capable of inducing remission (16).

In the advanced stages of TA, with significant stenosis or the occlusion of blood vessels, invasive treatment is performed as needed; angioplasty and stent placement are employed on specific arterial sites with stenosis of $<5 \mathrm{~cm}$, while more severe cases are more effectively treated with open surgery, which has lower re-stenosis rates and improves long-term survival; pre- and post-operative immunosuppression is crucial (17).

\section{Case report}

The patient presented herein was a 40 -year-old female with a history of tuberculosis and treatment for pleural effusion 8 years prior, and a urinary tract infection with Klebsiella, presented at the local county hospital (Ramnicu-Valcea County Hospital, Ramnicu-Valcea, Romania) with left hemiplegia and left central facial paresis. Computer tomography revealed cortico-subcortical ischemia in the territory of the right medial cerebral artery (MCA).

Upon admission to the Neurology Ward at Colentina Hospital (Bucharest, Romania), the patient was alert, oriented, unable to ambulate, in no acute distress, with a blood pressure (BP) of $130 / 80 \mathrm{mmHg}$ in the left arm, 70/50 $\mathrm{mmHg}$ in the right arm, pulse of $50 \mathrm{bpm}, \mathrm{O}_{2}$ saturation of $96 \%$ in room air; facial asymmetry with left facial paresis, no motor function of the left arm, left leg exhibited dorsiflexion of the foot, could maintain a seated position, deglutition was intact but with Foley catheter for neurological bladder, positive Babinski sign and Hoffman reflex on the left side, sensory deficit on the left side; and a Barthel index of 5. Echocardiography revealed pericardial effusion, normal systolic and diastolic function with an ejection fraction of $60 \%$.
An AngioCT revealed the occlusion of the right subclavian and common carotid arteries, and stenosis of the left common carotid artery. Temporal artery biopsy confirmed the diagnosis of TA. Bloodwork analysis revealed the following: Normal levels of electrolytes, lipid profiles, bilirubin, creatine kinase, uric acid, creatinine, amylase, alkaline phosphatase, fibrinogen, anti-pANCA, anti-Ro, anti-double cDNA, cardiolipin IgG and $\operatorname{IgM}, \beta$-2 glycoprotein $\operatorname{IgG}$, lupus anticoagulant and protein $\mathrm{C}$; increase levels of liver function tests-ALT 112 UI [upper limit of normal (ULN) 31]; AST 42 (ULN 32); ESR 29-62 mm/h (ULN 20) leucocytosis $13 \times 10^{6} / \mathrm{ml}$ (ULN 10), anti-nuclear antibodies (ANA) 33 (ULN 20) UI/ml, INR 1,31 (ULN 1,14); and decreased levels of hematocrit $11.3 \mathrm{~g} / \mathrm{dl}$ [lower limit of normal (LLN) 11.7] protein S (46\%, LLN 60\%).

The patient was placed on Medrol $16 \mathrm{mg}$ bid, statin and a proton pump inhibitor, fraxiparine 0.6 UI bid. After approximately 1 week on this treatment, during which the patient regained bladder control and the Foley catheter was taken out, the patient was complaining about left-sided chest pains, referring to the left arm, and she was transferred for urgent coronary angiography.

Upon admission to the Angiomedica Hospital (Bucharest, Romania) the patient exhibited diminished subclavicular pulse on the right side, pulses present in both the left and right radio-ulnar arteries, as well as both feet.

The carotid Doppler revealed the occlusion of common carotid arteries bilaterally, as well as the occlusion of the right external and internal carotid arteries, as well as the right vertebral artery; the left internal and external carotid arteries were patent, probably through the Willis circle.

The coronary angiography revealed no coronary stenosis and confirmed the occlusion of the common carotid arteries (Fig. 1A-C; angiographs illustrating the occlusion of the carotids).

Since endovascular treatment was excluded, the patient was administered intravenous medication consisting of an original combination of medicines which included dexamethasone $8 \mathrm{mg}$ (18) and the patient was planned to be discharged the following morning in a long-term neurological recovery institution.

That morning the patient was serendipitously presented to the cardiovascular surgeon, Z.G., who was present by chance in the angiography suite, and after discussing the case with the interventional cardiologist and performing another vascular Doppler on the carotids, patient and family were informed on the surgical treatment and a decision was made to perform a left-to-right vascular bypass.

Informed consent was obtained from both the patient and the family prior to surgery; for the duration of the procedure (approximately $8 \mathrm{~h}$ ); conscious intravenous sedation was administered by the anesthesiologist, M.F.; approximately $2 \mathrm{~h}$ into the procedure, there was an episode of low blood pressure with the patient responding well to administration of Voluven solution.

The vascular bypass used venous grafts harvested by E.Q. and M.C. from the patient's both external saphenous veins and it involved performing a bypass between the left external carotid artery and the left subclavian artery in the inverted UZI technique, and a vascular bypass was performed as well to the right vertebral artery in the suboccipital triangle 


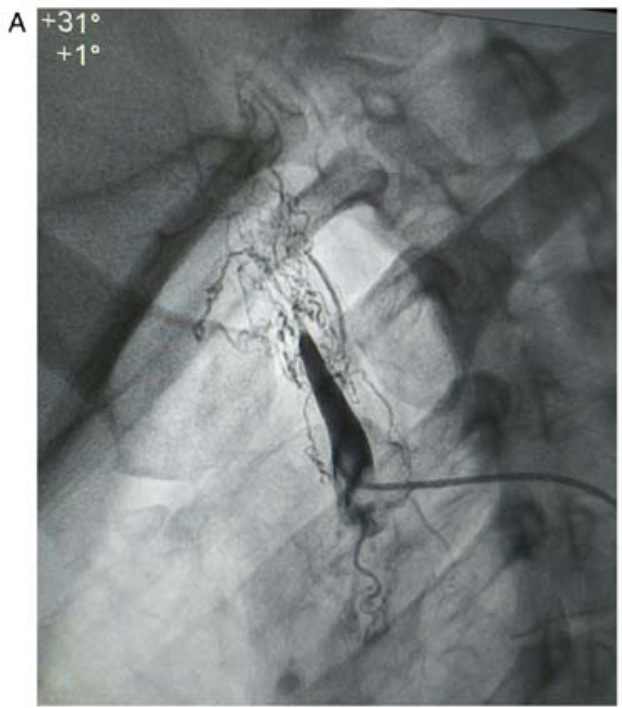

B
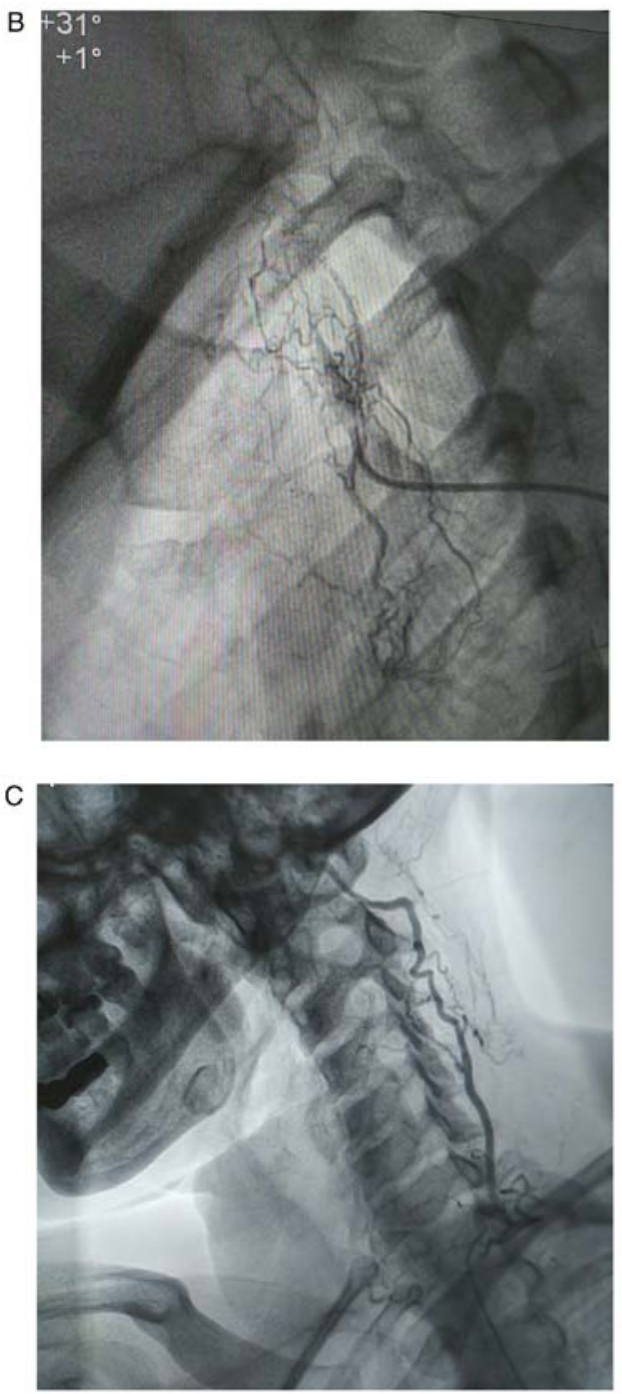

Figure 1. (A-C) Angiographs showing occlusions of the carotids.

(via tunneling). An image of the dissected suboccipital triangle was acquired (Fig. 2; intra-operative visualization of the right vertebral artery in the suboccipital triangle).

The left-right shunt patency was examined intra- and post-operatively with the presence of a pulse in the shunt,

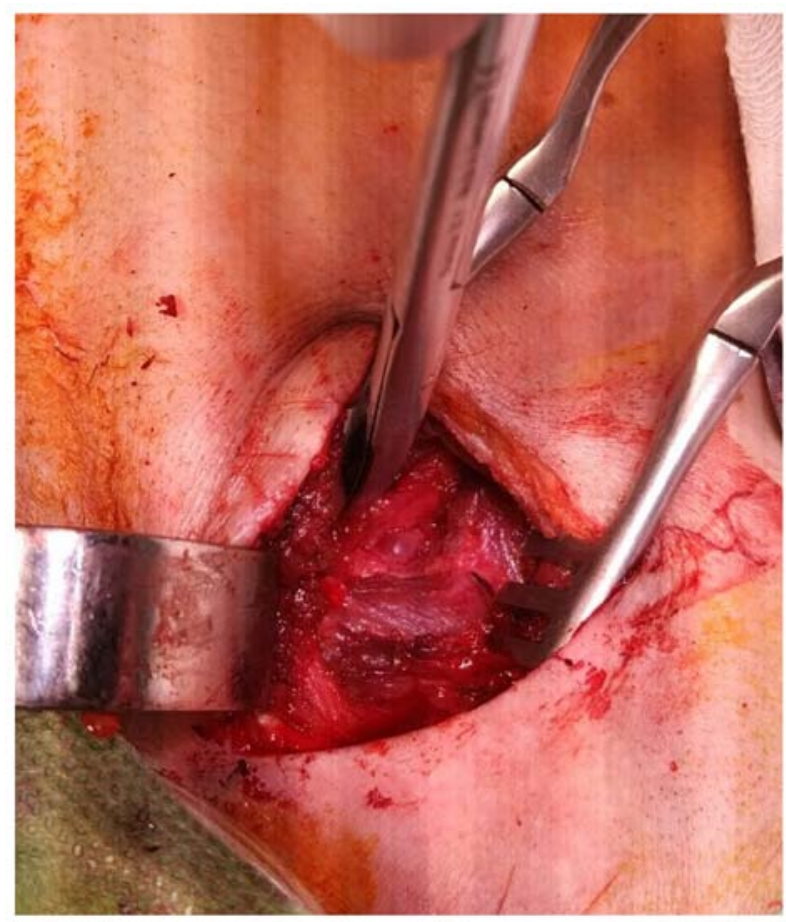

Figure 2. Intra-operative visualization of the right vertebral artery in the suboccipital triangle).

which can be palpated superficially, under the skin, in the anterior neck above the thyroid. Post-operatively, there were no new neurological deficits, no complications or adverse reactions.

Another intravenous neurotrophic combination, which included dexamethasone at $8 \mathrm{mg}$ was administered post-surgery, after which the patient presented with emesis as an adverse reaction; the patient then remained stable and was not in any distress, and was discharged in a neurologically stationary condition.

Notably, after approximately 1 week, the patient's family reported that the patient had improved motor skills on the left side, and at 3 months post-surgery the patient, was able to ambulate with a cane and was filmed doing so during a follow-up visit to the hospital. The motor improvement on the left arm was minimal.

At 18 months follow-up, the patient has maintained the motor abilities on the left leg and was able to perform independently the activities of daily living (Barthel index of 60). The patient is on Medrol $16 \mathrm{mg}$, and the persistent bradycardia is being evaluated for pacemaker stimulation.

\section{Discussion}

Even though this patient was not tested for the presence of 60-65 kDa HSP, nor for vascular endothelial antibodies, given the patient's history and the sequence of events, it is very likely that in this patient, the vasculitis was linked to the presence of mycobacterial infection. The criteria for TA present in this patient were age, pulse differential between left and right arms, decreased pulse and ultrasound and angiographic arterial stenosis. The left-sided chest pain for which the patient was referred for coronary angiograph was most likely due 
to ischemic conditions, either locally in the chest or referred neurological pain; other possible causes include presence of pericardial effusion and inflammation.

Due to the patient presenting with stroke, extensive laboratory workup (TNF- $\alpha$, IL-6, endothelial antibodies) was superseded by the need for initiation of corticoid therapy and anticoagulation, which was followed by a decrease of erythrocyte sedimentation rate from 62 to $29 \mathrm{mmHg}$ and improved bladder control during initial care at the referring hospital.

Treatment options. Even though the majority of patients with TA are treated with medication only, in the case of stenosed or occluded arteries, vascular intervention offers the most effective long-term treatment option, particularly for organ ischemia, hypertension and aneurysmal disease $(11,17,19,20)$.

Endovascular procedures (angioplasty and stents) are less invasive and have lower complication rates than open surgical procedures (vascular bypass). Moreover, newly developed eprolimus-covered stents have proven to be similar in therapeutic efficacy to coronary bypass surgery [EXCEL trial (21)], and they may also prove to be a good therapeutic option in the earlier stages of TA, where there is limited vascular damage.

However, in the long term, the restenosis rates for endovascular procedures are higher, approximately $50 \%$ at 5 years, and in some studies are as high as $80 \%(19,22)$ compared to surgical revascularization, after which vascular patency is preserved in approximately $80 \%$ of patients and the mean graft patency is preserved for 9.4 years.

A specific feature of TA is that it occurs at a younger age ( $<40$ years) and for this reason, long-term vascular patency becomes a critical issue when compared to atherosclerotic disease; another strong argument in favor of vascular surgery is the possibility of vessel dissection and/or rupture when baloon angioplasty is employed (19).

Considering the issue of severe early complications observed with vascular surgery (intracerebral hemorrhage, hyperperfusion syndrome and cardiac tamponade), as well as its superior long-term patency compared to endovascular procedures (23), endovascular intervention is recommended in the case of stenotic lesions with a length of $<5 \mathrm{~cm}$, and respectively for longer occlusive lesions, bypass surgery; these authors observed at 39 months a mean restenosis rate of 53\% for endovascular procedures and $12.5 \%$ for vascular bypass; the most frequent symptom in these patients was brain ischemia (23).

To identify the risk factors for complications of vascular procedures, a clinical study on 66 patients with TA who had 119 vascular procedures (arm claudication was the most frequent symptom) was performed (24); in these patients, there was a higher risk of complications and restenosis observed in patients with hypertension, dyslipidemia and patients treated with higher doses of steroid medications.

Active vs. inactive disease. For this patient presenting with stroke, prompt revascularization proved to be an effective therapeutic option irrespective of the TA activity status; however, it was observed that vascular procedures, surgical or endovascular, have optimal results when performed during inactive disease (11); the administration of steroids or immunosuppressants before the procedures improved vascular inflammation, deterioration of elastic fibers and aneurysm formation; during active disease, the risk of restenosis, dissection and complications is increased, and in commonly, interventions are performed after active inflammation is controlled (19).

Disease activity in TA can be inferred from increased levels of serum IL- 6 and TNF $\alpha$ and a higher uptake of 18F-Fluorodeoxyglucose [(18)F-FDG] in the arterial wall $(25,22)$; even so, distinguishing between systemic and vascular inflammation is difficult and various evaluations have been suggested towards this end: The Indian Takayasu Clinical Activity Score (12), while other authors have recommended direct imaging of vessel wall thickening (ultrasound, CT, angiogram) or positron emission tomography computerized tomography (PET CT) (11).

Prognosis and survival. Based on the literature cited and due to the lack of early complications and at 18 months, the medium- and long-term prognostic for this patient was good. Supporting this assessment, is a retrospective review (26) which cites a 92-year-old patient who survived 23 years after vascular bypass without immunotherapy, as well as an analysis of 106 consecutive patients with TA (20), in whom there was a $73.5 \%$ survival rate at 20 years (congestive heart failure was most common problem), and anastomotic aneurysm had a cumulative incidence of $13.8 \%$ at the 20 -year follow-up, for which regular follow-up imaging was recommended and concluded that for patients with occluded arteries, surgery is the optimal long-term option.

\section{Acknowledgements}

The authors would like to thank Dr Roxana Zmarandescu from Colentina Hospital for referring the patient presented in this case report.

\section{Funding}

No funding was received.

\section{Availability of data and materials}

Data sharing is not applicable to this article, as no datasets were generated or analyzed during the current study.

\section{Authors' contributions}

ZG was involved in the conception and design of the study, and in the planning and performing of the surgical procedures, data collection and obtaining surgical images. FS was involved in the conception and design of the study, and in the writing of the manuscript, data collection and image processing; DAS was involved in the conception and design of the study, and in data analysis and reviewing/revisions; GL was involved in the conception and design of the study, and in data analysis and reviewing/revisions. MC was involved in the conception and design of the study, and in data collection from surgical procedures. EQ was involved in the conception and design of the study, and in data collection from surgery, including 
images. MF was involved in the conception and design of the study, and in data collection from surgery. SK was involved in the conception and design of the study, and in data analysis and reviewing/revisions. All authors have read and approved the final manuscript.

\section{Ethics approval and consent to participate}

Informed consent was obtained from both the patient and the family prior to surgery.

\section{Patient consent for publication}

The patient provided consent prior to publication for her data to become public.

\section{Competing interests}

DAS is the Managing Editor of the journal, but had no personal involvement in the reviewing process, or any influence in terms of adjudicating on the final decision, for this article. The other authors declare that they have not competing interests.

\section{References}

1. Russo RAG and Katsicas MM: Takayasu arteritis. Front Pediatr 6: 265, 2018.

2. Arend WP, Michel BA, Bloch DA, Hunder GG, Calabrese LH, Edworthy SM, Fauci AS, Leavitt RY,Lie JT, Lightfoot RW Jr, et al: The American college of rheumatology 1990 criteria for the classification of Takayasu arteritis. Arthritis Rheum 33: 1129-1134, 1990.

3. Vaideeswar P and Deshpande JR: Pathology of Takayasu arteritis: A brief review. Ann Pediatr Cardiol 6: 52-58, 2013.

4. Arnaud L, Haroche J, Mathian A, Gorochov G and Amoura Z: Pathogenesis of Takayasu's arteritis: A 2011 update. Autoimmun Rev 11: 61-67, 2011.

5. Kong X, Sun Y, Ma L, Chen H, Wei L, Wu W, Ji Z, Ma L, Zhang Z, Zhang Z, et al: The critical role of IL-6 in the pathogenesis of Takayasu arteritis. Clin Exp Rheumatol 34 (Suppl): S21-S27, 2016.

6. Wu G, Mahajan N and Dhawan V: Acknowledged signatures of matrix metalloproteinases in Takayasu's arteritis. Biomed Res Int 2014: 827105, 2014.

7. Tripathy NK, Chandran V, Garg NK, Sinha N and Nityanand S: Soluble endothelial cell adhesion molecules and their relationship to disease activity in Takayasu's arteritis. J Rheumatol 35 1842-1845, 2008.

8. Kumar Chauhan S, Kumar Tripathy N, Sinha N, Singh M and Nityanand S: Cellular and humoral immune responses to mycobacterial heat shock protein-65 and its human homologue in Takayasu's arteritis. Clin Exp Immunol 138: 547-553, 2004.

9. Chauhan SK, Tripathy NK and Nityanand S: Antigenic targets and pathogenicity of anti-aortic endothelial cell antibodies in Takayasu arteritis. Arthritis Rheum 54: 2326-2333, 2006.

10. Espinoza JL, Ai S and Matsumura I: New insights on the pathogenesis of Takayasu arteritis: Revisiting the microbial theory. Pathogens 7: 73, 2018.
11. Misra DP, Wakhlu A, Agarwal V and Danda D: Recent advances in the management of Takayasu arteritis. Int J Rheum Dis 22 (Suppl): 60-68, 2019.

12. Keser G, Aksu K and Direskeneli H: Takayasu arteritis: An update. Turk J Med Sci 48: 681-697, 2018.

13. Ataş N, Varan Ö, Babaoğlu H, Satiş H, Bilici Salman R and Tufan A: Certolizumab pegol treatment in three patients with Takayasu arteritis. Arch Rheumatol 34: 357-362, 2019.

14. Shao N, Jia H, Li Y and Li J: Curcumin improves treatment outcome of Takayasu arteritis patients by reducing TNF- $\alpha$ : A randomized placebo-controlled double-blind clinical trial. Immunol Res 65: 969-974, 2017.

15. Shi G, Hua M, Xu Q and Ren T: Resveratrol improves treatment outcome and laboratory parameters in patients with Takayasu arteritis: A randomized double-blind and placebo-controlled trial. Immunobiology 222: 164-168. 2017.

16. Matsuyama A, Sakai N, Ishigami M, Hiraoka H and Yamashita S: Minocycline for the treatment of Takayasu arteritis. Ann Intern Med 143: 394-395, 2005.

17. Mason JC: Surgical intervention and its role in Takayasu arteritis. Best Pract Res Clin Rheumatol 32: 112-124, 2018.

18. Stancioiu F and Makk R: Post-stroke recovery of motor function with a new combination of medicines-A pilot study. EJMO 3: 167-181, 2019.

19. Jeong HS, Jung JH, Song GG, Choi SJ and Hong SJ: Endovascular balloon angioplasty versus stenting in patients with Takayasu arteritis: A meta-analysis. Medicine (Baltimore) 96: e7558, 2017.

20. Miyata T, Sato O, Koyama H Shigematsu H and Tada Y: Long-term survival after surgical treatment of patients with Takayasu's arteritis. Circulation 108: 1474-1480, 2003.

21. Stone GW, Sabik JF, Serruys PW, Simonton CA, Généreux P, Puskas J, Kandzari DE, Morice MC, Lembo N, Brown WM III, et al: Everolimus-eluting stents or bypass surgery for left main coronary artery disease. N Engl J Med 375: 2223-2235, 2016.

22. Perera AH, Youngstein T, Gibbs RG, Jackson JE, Wolfe JH and Mason JC: Optimizing the outcome of vascular intervention for Takayasu arteritis. Br J Surg 101: 43-50, 2014.

23. Kim YW, Kim DI, Park YJ, Yang SS, Lee GY, Kim DK, Kim K and Sung K: Surgical bypass vs endovascular treatment for patients with supra-aortic arterial occlusive disease due to Takayasu arteritis. J Vasc Surg 55: 693-700, 2012.

24. Labarca C, Makol A, Crowson CS, Kermani TA, Matteson EL and Warrington KJ: Retrospective comparison of open versus endovascular procedures for Takayasu arteritis. J Rheumatol 43: 427-432, 2016

25. Arraes AE, de Souza AW, Mariz HA, Silva NP, Torres IC, Pinto PN, Lima EN and Sato EI: (18)F-Fluorodeoxyglucose positron emission tomography and serum cytokines and matrix metalloproteinases in the assessment of disease activity in Takayasu's arteritis. Rev Bras Reumatol Engl Ed 56: 299-308, 2016.

26. Yoshida M,Zoshima T, Hara S, Mizushima I, Fujii H, Yamada K, Sato Y, Harada K and Kawano M: A long-term survival after surgical treatment for atypical aortic coarctation complicating Takayasu arteritis with inactive disease at the diagnosis: An appropriately treated autopsy case. Intern Med 58: 2241-2246, 2019. 